CAHIERS DE

NARRATOLOGIE
Cahiers de Narratologie

Analyse et théorie narratives

$10.1 \mid 2001$

La voix narrative

\title{
Brillante porvenir de Vicente Aranda : l'équivocité du discours au service de la liberté intellectuelle
}

Jean-Paul Aubert

\section{(2) OpenEdition}

1 Journals

\section{Electronic version}

URL: http://journals.openedition.org/narratologie/6921

DOI: 10.4000/narratologie.6921

ISSN: 1765-307X

\section{Publisher}

LIRCES

\section{Printed version}

Date of publication: 1 January 2001

Number of pages: 103-111

ISBN: 2914561032

ISSN: 0993-8516

\section{Electronic reference}

Jean-Paul Aubert, "Brillante porvenir de Vicente Aranda : I'équivocité du discours au service de la liberté intellectuelle", Cahiers de Narratologie [Online], 10.1 | 2001, Online since 16 October 2014, connection on 24 February 2021. URL: http://journals.openedition.org/narratologie/6921 ; DOI: https://doi.org/ 10.4000 /narratologie.6921 


\title{
BRILLANTE PORVENIR DE VICENTE ARANDA : L'ÉQUIVOCITÉ DU, DISCOURS AU SERVICE DE LA LIBERTÉ INTELLECTUELLE
}

\author{
Jean-Paul AUBERT \\ Université de Nice-Sophia Antipolis
}

La filmographie du réalisateur espagnol Vicente Aranda est riche de vingt longs-métrages. Nous nous intéresserons ici au premier d'entre eux, l'un des moins connus aussi : Brillante porvenir (1964), fruit d'une collaboration entre Román Gubern, Ricardo Bofill et Vicente Aranda. Le film nous fait découvrir l'itinéraire d'un jeune aide-architecte provincial interprété par Germán Cobos à qui l'on propose soudainement de travailler dans un prestigieux cabinet d'architecture de Barcelone. Il y fait la rencontre de Lorenzo (Arturo López) qui le prend en main, lui sert de guide au sein de la haute société de la capitale catalane, et lui offre même un appartement où passer l'été. Lorenzo a une sœur, Montse (Serena Vergano), dont Antonio s'éprend avant de s'apercevoir qu'il doit faire face à un rival en la personne de López (José María Angelat), homme riche et puissant. Montse, quant à elle, n'a qu'un rêve : fuir l'Espagne et partir pour Paris. López, aidé de Lorenzo, tente par la persuasion puis par la menace de faire en sorte qu'Antonio renonce à Montse. Ils semblent échouer puisque l'avant-dernière séquence du film montre un Antonio triomphant, montant dans une belle décapotable conduite par la jeune femme bien décidée à partir pour Paris. Mais soudain la voiture s'arrête et Antonio, qui prend conscience de n'avoir fait que servir les desseins égoïstes de Montse, descend et marche en direction de la caméra. Tout paraît indiquer que le film va s'achever sur cette image gelée qui marque la rupture entre les deux amants quand, dans un dernier retournement de situation, apparaît en gros plan, face à la caméra le visage de Montse qui s'adresse au spectateur en ces termes : 
- «Tengo que decirles unas palabras. Antonio ha contado su historia con muchas inexactitudes. Pero la mayor de todas es este final. Antonio vino conmigo a París. Esto es todo. »

C'est plus particulièrement cette dernière séquence, dont la censure exigea la suppression, qui va retenir notre attention. Elle est porteuse d'un message dont le déchiffrement oblige à en mesurer la portée dans le contexte idéologique et artistique de l'époque. Elle marque tout d'abord une rupture dans un récit fondé sur le point de vue d'Antonio. Elle pose en outre le problème, par ailleurs souvent débattu, du regard à la caméra, dénonciateur de la fiction comme leurre. Enfïn, l'intervention de Montse, révélatrice d'une supercherie, souligne combien la vérité est fragile et le réel toujours plus complexe qu'il n'y paraît.

\section{1 - Le point de vue d'Antonio}

Dans son intervention, Montse rappelle au spectateur distrait une donnée essentielle : le récit n'a cessé de privilégier le point de vue subjectif d'Antonio. Précisons qu'il s'agit d'un point de vue à la fois perceptif et narratif. Perceptif car le film multiplie les images subjectives (le spectateur voit ce que voit Antonio) ou semi-subjectives (la caméra est placée derrière le personnage de sorte qu'une partie du corps de celui-ci apparaît en amorce), ainsi que les effets de son subjectif. Le point de vue subjectif est également narratif. En effet, d'emblée, le film se présente comme un récit à la première personne. La première séquence de pré-générique fait entendre la voix-off d'un narrateur qui n'est autre qu'Antonio :

- « 'Muy señor mío'. Todos los días, durante ocho horas 'Muy señor mío'. Mis compañeros de trabajo. O lo que conocía de ellos. Y esa casa extraña donde uno come y duerme. Y estudiar a solas durante horas y horas. Ese paseo melancólico y vacío. Esa aventura inútil. Así fue mi vida durante varios años hasta que un día, inesperadamente, una puerta se abrió. »

L'emploi du passé simple - « una puerta se abrió » - souligne le fait que nous sommes en présence d'une histoire révolue dont le narrateur est supposé tout savoir. Il semble en outre 
conscient de son statut de narrateur. Il est, pour reprendre les mots de François Jost « le premier spectateur de la visualisation qu'il nous propose $\gg 1$. Le discours se fait commentaire des plans qui se succèdent et nous montrent les collègues de travail du narrateur, celui-ci en train de déjeuner ou à sa table de travail, ou encore errant sans but. Les phrases courtes telles que "Mis compañeros de trabajo. » et l'emploi des déictiques " ese », " esa » désignent une image que le narrateur sait présente. L'image incarne ce que la parole évoque. La voix qui raconte l'histoire ayant déjà eu lieu, est dotée d'une sorte d'autorité sur l'image et en retour, l'image authentifie le discours prononcé. Certes, la voix-off du narrateur ne se fait entendre que dans l'incipit mais on retiendra avec Nicole de Mourgues que la séquence inaugurale d'un film en est comme la «matrice $»^{2}$. De sorte que si la porte qui s'ouvre à l'issue du pré-générique est bien une porte de sortie pour Antonio, elle est aussi une porte d'entrée pour le spectateur qui, guidé par le narrateur, peut se laisser entraîner dans le récit qui va suivre. Et si la voix-off par la suite se tait, l'instance narratrice mise en place par le pré-générique domine l'ensemble du récit. En outre, si le spectateur, au cours du film, avait oublié qu'Antonio était à l'origine du récit, Montse le lui rappelle dans l'ultime séquence : "Antonio ha contado su historia $[\ldots] »$.

\section{2 - Le mensonge}

L'intervention de la jeune femme est dotée d'un évident statut métatextuel. Sa place aux confins du récit, sa valeur de commentaire du texte filmique qui la précède font de cette séquence une préface selon la définition qu'en donne Genette $^{3}$. Cette valeur métatextuelle du dernier plan est également soulignée par le fait que Montse n'apparaît pas en situation diégétique. Le gros plan dont elle fait l'objet ne

${ }^{1}$ François JOST, «Règles du Je », Iris, n ${ }^{\circ}$, $2^{\circ}$ semestre 1988, p. 111.

2 Nicole MOURGUES (De), Le Générique de film, Paris, Méridiens Klincksieck, 1994, p. 77.

${ }^{3}$ Gérard GENETTE, Seuil, Paris, Le Seuil, 1987, p. 150. 
permet pas d'apprécier un éventuel décor qui l'inclurait dans le récit. Cette dernière séquence constitue donc une rupture formelle avec le reste du film ; une rupture consommée dans les propos de la jeune femme mettant en cause la véracité du récit proposé par Antonio.

Dans un article pionnier sur la question des films à la première personne, Jean-Pierre Chartier souligne combien le récit est crédibilisé par l'emploi du « je $»^{4}$. Mais l'emploi de la première personne introduit également une part de subjectivité. On n'a pas affaire ici à un "je » témoin, au fond bien proche du «il », mais à un « je » qui nous raconte sa propre histoire. C'est ce que souligne l'intervention de Montse dont la première conséquence est d'ébranler fortement la crédibilité du narrateur qui n'est porteur que d'une vérité relative à sa perception. Le coup est d'autant plus rude pour le spectateur qu'il s'est identifié au personnage d'Antonio et que la révélation inattendue de Montse, qui intervient brutalement et in extremis, remet en cause l'ensemble du récit. Deux vérités entrent donc en conflit. Il reste à déterminer qui des deux ment. La tâche n'est pas facile pour le spectateur qui doit choisir entre la version d'une manipulatrice et celle d'un personnage surpris à plusieurs reprises en flagrant délit de tromperie. En effet, Antonio s'est fait passer pour architecte, lui qui n'est qu'aide- architecte. Modeste employé de bureau de province, il n'a pas hésité non plus à revêtir, à l'occasion d'une soirée, un smoking de location pour donner l'illusion qu'il appartient à la haute bourgeoisie catalane. Ces deux détails qui, sur le moment, avaient pu être négligés par le spectateur, revêtent à la lumière des propos de Montse, une singulière importance. Ne suggéraient-ils pas déjà la possible duplicité du personnage ? Le spectateur est donc invité à opérer un retour réflexif sur le récit qui vient de se dérouler sous ses yeux et à réévaluer les informations qui lui ont été données. Pour autant, la conclusion du film le frustre d'une solution satisfaisante à l'intrigue. Antonio est-il oui ou non allé à Paris avec Montse? Il n'est désormais plus possible de

4 Jean-Pierre CHARTIER, « Les 'Films à la première personne' et l'illusion de réalité au cinéma ", La Revue du cinéma, janvier 1947, p. 35 . 
répondre avec certitude à cette question. La tension née de cette interrogation se résout dans une indécision. Au fond, il importe peu d'attribuer la paternité du mensonge. L'essentiel est ailleurs dans cette véritable stratégie de brouillage de la vérité qui passe par la confrontation de vérités relatives et la mise en évidence d'un réel complexe et difficile à déchiffrer. Ajoutons qu'Antonio et Montse, manipulateurs potentiels, évoluent dans un univers où règnent le faux-semblant et le mensonge. Ainsi, lors d'une réception, le père de Lorenzo fait admirer fièrement une fontaine d'apparence classique mais qui s'avère en fait de construction récente. Les nombreux équipements modernes dont s'entourent les personnages sont soit inutiles, soit défectueux. Nous voilà plongés dans un monde fait d'incertitudes où il est bien difficile de démêler le vrai du faux.

En cela Brillante porvenir s'inscrit dans le cadre des préoccupations esthétiques et philosophiques des années soixante. En 1962, a été publié en Italie l'ouvrage d'Umberto Eco, L'CEuvre ouverte, qui caractérise l'œuvre d'art comme un "message fondamentalement ambigu » et fait de l'indéterminé "une catégorie du savoir ${ }^{5}$. La réflexion théorique d'Umberto Eco ne fait qu'accompagner la remise en cause des certitudes et des vérités toutes faites, opérée par une nouvelle génération de créateurs qui entendent donner à la narration un nouveau statut ainsi défini par Gilles Deleuze :

[...] la narration cesse d'être véridique, c'est-à-dire de prétendre au vrai, pour se faire essentiellement falsifiante ${ }^{6}$.

On ne peut s'étonner dès lors de voir la supercherie mise à l'honneur. Antonio et Montse ne sont que deux menteurs de plus dans la longue liste des falsificateurs, entourloupeurs et faussaires qu'affectionnent les tenants du nouveau cinéma. Le premier opus de Vicente Aranda avait notamment été précédé par un film intitulé Les Plus belles escroqueries du

5 Umbcrto ECO, L'Euvre ouverte, (lère éd. : Milan, Bompiani, 1962), Paris, Seuil, 1965, p. 9 et p. 30.

${ }^{6}$ Gilles DELEUZE, Cinéma 2. L'image-temps, Paris, Minuit, 1985, p. 171. 
monde, (1963) composé de plusieurs longs-métrages signés Hirimochi Horikawa, Roman Polanski, Ugo Gregoretti, Claude Chabrol et Jean-Luc Godard. On se souvient qu'avant eux, Orson Welles avait fait de la manipulation et de l'escroquerie des thèmes cinématographiques. Le premier film de Vicente Aranda assimile donc cet héritage artistique et philosophique. Il rejoint par ailleurs les principes de la narration objective dont s'emparent en Espagne des écrivains tels que Juan Goytisolo. On découvre transposée dans ce film cette même conception du récit qui balaye l'omniscience narrative et, selon les mots de Geneviève Champeau, limite « la représentation à des points de vue particuliers excluant toute vérité absolue, tout savoir qui ne soit pas celui d'un sujet individuel $»^{7}$.

\section{3 - L'instance énonciatrice dévoilée et décrédibilisée}

Les quelques secondes du face à face final entre Montse et le spectateur et qui permettent à ce dernier d'éprouver la présence de la caméra, ont comme autre conséquence de dévoiler l'instance énonciatrice. On a beaucoup glosé sur la question du regard à la caméra. Il constitue, on le sait, une transgression des règles habituelles ${ }^{8}$. Le regard de Montse à la caméra relève du "contre-regard " théorisé par Pascal Bonitzer, celui qu'affectionnent Godard, Straub et un certain nombre d'auteurs avant-gardistes; il fixe et interpelle la communauté des spectateurs et révèle brutalement la nature fictionnelle de l'œuvre ${ }^{9}$. En effet, la première phrase que Montse prononce : "Tengo que decirles unas palabras ", s'adresse directement et sans ambiguïté au spectateur, véritablement interpellé selon une démarche distanciatrice que l'on est tenté de qualifier de brechtienne. La présence de l'énonciateur, si elle est dans cette dernière séquence explici-

7 Geneviève CHAMPEAU, Les Enjeux du réalisme dans les romans sous le franquisme, Madrid, Casa de Velázquez, 1993, p. 382.

8 Voir Roland BARTHES, «Droit dans les yeux ", L'Obvie et l'obtus, Paris, Le Seuil, 1982, p. 282; Marc Vernet, Figures de L'absence, Paris, Éditions de l'Étoile, 1988, pp. 9-25.

9 Pascal BONITZER, «La notion de plan et de sujet du cinéma », Cahiers du cinéma, $\mathrm{n}^{\circ} 275$, avril 1977, p. 45. 
tement dévoilée, se lit à d'autres instants du film et notamment dans les références intertextuelles dont il est émaillé. On ne peut en effet s'empêcher de rapprocher Brillante porvenir de La Dolce vita (1959) de Federico Fellini ${ }^{10}$. Les deux films coïncident dans la mise en scène d'une bourgeoisie - ou petite-bourgeoisie - entièrement gagnée par la superficialité et l'oisiveté. La mise en scène de la rupture entre les deux amants, fausse conclusion de Brillante porvenir, n'est pas sans rappeler une séquence du chef-d'œuvre fellinien, celle de la dispute entre Marcello et sa compagne. Hommage au maître, clin d'œil au cinéphile, cette séquence souligne, plus discrètement sans doute que ne le fait le regard à la caméra mais non moins clairement cependant, la présence d'un énonciateur, manipulateur à ses heures. C'est également sur le compte de cette instance implicite qu'il faut mettre cette amusante allusion à l'un des scénaristes du film. Dans la cinquième séquence du film, Antonio qui dicte une lettre, précise : « arquitecto : don Ricardo Bofill Levi, este Levi con 'v'».

L'apparition de Montse ne se contente pas de dévoiler avec fracas l'instance énonciatrice, elle la décrédibilise. Bien que le film privilégie les effets de caméra subjective ou semisubjective, nous l'avons signalé au début de ce travail, il n'élimine évidemment pas les plans objectifs sur le personnage. Or à aucun moment, on ne constate de distorsion entre images objectives et images subjectives. Au contraire, les premières viennent corroborer les secondes. Par conséquent, le discours de Montse fait de la caméra et de l'énonciateur les complices des falsifications du narrateur. $\mathrm{Si}$, comme le dit Montse, la version d'Antonio selon laquelle il ne l'a pas accompagnée à Paris est mensongère, alors le plan objectif, montrant le jeune homme qui descend de voiture et s'en éloigne, est de fait discrédité. Le discours de vérité qui est censé caractériser l'image objective est ici battu en brèche.

10 On pourrait encore rapprocher Brillante porvenir du chef d'œuvre de Robert Wiene, Le Cabinet du Dr Caligari (1919) et souligner le mimétisme entre le film d'Aranda et l'œuvre de Michelangelo Antonioni. 
Tout du moins, le discours de Montse jette-il la suspicion sur l'instance énonciatrice.

Ce n'est certes pas la seule tromperie dont l'énonciateur se serait rendu «coupable». Le plagiat du film de Fellini auquel il se livre relève également d'une volonté de falsification". Supercherie encore la pratique qui consiste à s'inspirer, sans le signaler, d'un roman, en l'occurrence ici Gatsby le magnifique de Scott Fitzgerald. Et l'on pourrait également mettre sur le compte de cette volonté de brouiller le sens, l'ambiguïté teintée d'ironie d'un titre qui est également imputable à l'instance énonciatrice.

\section{Épilogue}

Après avoir fait de la vérité une valeur relative et pour ainsi dire inaccessible, voici que le film invite à la méfiance à l'égard des images qui se donnent pour vraies. On comprend dès lors que la portée subversive de la dernière séquence de Brillante porvenir n'ait pas échappé au censeur. La commission chargée d'examiner le scénario exige sa suppression ${ }^{12}$. Le réalisateur s'entête. La Junta de Clasificación y Censura, qui, quelques mois plus tard, doit se prononcer sur le film réalisé, confirme l'interdiction de sa diffusion en l'état. Aux dires de l'un des membres de la commission «el plano final [...] da la nota de cinismo y amoralidad que hace negativa la tesis posible de la película »13. C'est donc un film amputé de son dernier plan qui sortira sur les écrans en 1964. Mais les ciseaux du censeur ne pourront rien finalement contre l'obstination du réalisateur. Plus de dix ans après, Aranda prend sa revanche et procède au remontage du film. C'est finalement dans sa version intégrale que l'on peut

11 Lire à ce sujet Jean-Claude SEGUIN, «Mujeres/The Women : le référentiel ", Postmodernité et écriture narrative dans l'Espagne contemporaine, Grenoble, 1996, pp. 273-288.

12 Session de la «Comisión delegada para censura de guiones cinematográficos » du 4 septembre 1963, Archivo central del Ministerio de Educación y Cultura, C/26 537 ; Exp. : 171-63.

13 Session de la Junta de Clasificación y censura du 9 juillet 1964 , Archivo central del Ministerio de Educación y cultura, C/54 509; Exp. : 31421. 
aujourd'hui visionner Brillante porvenir à la Filmoteca Española. Cette véritable guerre d'usure qui a opposé créateur et censeur autour de ce dernier plan en souligne l'importance. Il invite le spectateur à se méfier de la vérité univoque, dénonce la présence d'une instance énonciatrice implicite et, jouant de l'hétérogénéité entre le discours et l'image, met en doute la capacité de cette dernière à retranscrire la réalité. On comprend qu'un régime, qui, à grand renfort de propagande, célébrait "vingt-cinq années de paix » ne pouvait tolérer ce discours ambigu et pourfendeur de vérités toutes faites. 